\title{
Desenvolvendo práticas investigativas no Ensino Médio: o uso de um Objeto de Aprendizagem no estudo da Força de Lorentz ${ }^{+*}$
}

José Mauro Sousa ${ }^{1}$

E. E. Prof ${ }^{a}$. Maria Amália de Magalhães Turner

E. E. Joaquim Vilela de Oliveira Marcondes

Guaratinguetá - SP

Ana Paula dos Santos Malheiros ${ }^{2}$

Departamento de Educação

Universidade Estadual Paulista Júlio de Mesquita Filho

São José do Rio Preto - SP

Newton Figueiredo ${ }^{3}$

Instituto de Física e Química - Universidade Federal de Itajubá

Itajubá - MG

\section{Resumo}

Este artigo apresenta uma experiência de ensino na qual utilizamos o Objeto de Aprendizagem (OA) "Movimentos de Cargas num Campo Magnético", durante o estudo de conceitos relacionados à Força de Lorentz em uma turma da terceira série do Ensino Médio, em uma escola pública no interior do estado de São Paulo. Ao longo dessa experiência, investigamos as possíveis contribuições do OA para o ensino de conceitos de Eletromagnetismo, a partir do desenvolvimento de atividades de natureza investigativa que contemplavam a utilização de tal objeto. $O$ estudo foi pautado no paradigma qualitativo e a análise dos resultados mostrou que as simulações proporcionadas pelo OA possibilitaram a visualização dos fenômenos físicos estudados, os quais, no caso do Eletromagnetismo, exigem maior capacidade de abstração para o seu entendimento. As atividades realizadas também favoreceram o desenvolvi-

\footnotetext{
${ }^{+}$Developing investigative practices in High School: the use of a Learning Object to study the Lorentz force

* Recebido: agosto de 2014.

Aceito: junho de 2015.

1 E-mail: josemaurosousa@yahoo.com.br

2 E-mail: malheiros.anapaula@gmail.com

3 E-mail: newton@unifei.edu.br
} 
mento de determinadas habilidades relacionadas à competência de investigação e compreensão dos conceitos físicos. Além disso, uma maior interação entre os alunos e entre os alunos e o professor foi observada ao longo das aulas em que tal investigação foi desenvolvida.

Palavras-chave: Ensino de Física; Tecnologias de Informação e Comunicação; Visualização.

\begin{abstract}
This paper presents an educational experience in which we use the Learning Object (LO) "Cargo movements in a Magnetic Field" during the study of concepts related to the Lorentz force in a class of third year of high school in a public school in the state of São Paulo. Throughout this experience, we investigated the possible contributions of the LO for teaching concepts of Electromagnetism, from the development of investigative activities that contemplate the use of such an object. The study was guided by the qualitative paradigm and the analysis of the results showed that the simulations provided by $L O$ allowed the visualization of the studied physical phenomena which, in the case of Electromagnetism, requires greater capacity for abstraction to be understood. The activities also favored the development of certain skills related to the competence of researching and understanding of the physical concepts. In addition, greater interaction among students and between students and the teacher was observed during classes where this research was developed.
\end{abstract}

Keywords: Physics Teaching; Information and Communication Technologies; Viewing.

\title{
I. Introdução
}

O desenvolvimento da tecnologia verificado nas últimas décadas, bem como o crescente aumento do uso de recursos tecnológicos, principalmente no que diz respeito aos computadores e à internet, tem constituído importante fator de mudanças para diversos setores da sociedade. Entre essas mudanças, tem se destacado a utilização das Tecnologias de Informação e Comunicação (TIC) na área educacional, sobretudo no ensino de Ciências e, no ensino de Física, de modo particular.

Embora ainda sejam poucos os trabalhos que tratam da adoção de práticas pedagógicas no Ensino Médio que contemplem o uso das TIC durante as aulas de Física, nota-se que 
boa parte das pesquisas relata a utilização das tecnologias computacionais, envolvendo, principalmente, atividades com modelagem, animações ou simulações (ROSA, 1995; ARAÚJO; VEIT, 2004; MARTINS et al., 2011). Além disso, também é comum o seu uso associado a outros recursos computacionais, como as multimídias ${ }^{4}$ e os ambientes virtuais de aprendizagem (AVA) ${ }^{5}$.

Recentemente, o termo objetos de aprendizagem (OA) tem sido adotado com certa frequência ao se fazer referência aos diferentes materiais digitais de apoio à aprendizagem, sendo as simulações computacionais de experimentos os OA que mais têm ganhado destaque no ensino de Física (MIRANDA et al., 2011).

O presente artigo constitui o recorte de uma pesquisa pautada no paradigma qualitativo (TRIVIÑOS, 2009) e tem por objetivo investigar as potencialidades e as contribuições dos OA durante a abordagem de conceitos de Física para alunos de Ensino Médio, a partir do uso de simulações em atividades investigativas nas aulas de Eletromagnetismo. Para isso, descrevemos uma experiência na qual foi utilizado o OA "Movimentos de Cargas num Campo Magnético" ao longo do estudo de conceitos relacionados à Força de Lorentz em uma turma da terceira série do Ensino Médio de uma escola pública do interior de São Paulo.

Tal experiência foi desenvolvida a partir de apontamentos realizados sobre as possibilidades do uso de OA para o ensino de Ciências, dado o seu caráter dinâmico capaz de influenciar positivamente a aprendizagem do aluno nas mais diferentes situações (SANTOS NETO; SASSERON; PIETROCOLA, 2009). Aliado a isso, também foi considerado o fato de ser o Eletromagnetismo um dos temas de maior dificuldade de aprendizagem pelos estudantes, sobretudo diante dos obstáculos encontrados "na visualização espacial das interações entre as grandezas físicas e nas relações matemáticas que envolvem estas grandezas" (PAZ, 2007, p. 25). Assim, partindo-se do pressuposto de que a utilização de um OA possa favorecer a superação de tais dificuldades durante o ensino de conceitos de Eletromagnetismo, buscouse identificar se tal hipótese se verificava na prática durante as aulas de Física em uma escola pública.

Neste artigo, abordamos inicialmente o potencial dos OA nas atividades experimentais de Física. Em seguida, discutimos questões relacionadas ao papel da visualização no Ensino de Física e descrevemos o contexto do estudo, assim como os procedimentos metodológicos utilizados. Na sequência, apresentamos as atividades desenvolvidas e a análise das mesmas. Por fim, tecemos algumas considerações em torno dos principais aspectos observados ao longo de nossa pesquisa.

\footnotetext{
4 Araújo e Veit (2004) utilizam o termo "recursos multimídias", ao se referir a uma variedade de elementos, tais como textos, imagens, sons, vídeos, animações e simulações, organizados em módulos e inter-relacionados por links, permitindo ao aluno um caminho a ser seguido conforme o seu interesse.

5 Ambiente hipermídia na Web composto por simulações, modelagens e visualizações, sendo desenvolvido com o objetivo de proporcionar recursos que contemplem a aprendizagem coletiva e individual (ALMEIDA et al., 2001).
} 


\section{Simulações computacionais de experimentos físicos: as potencialidades oferecidas por este tipo de OA}

As atividades experimentais apresentam-se como uma prática capaz de contribuir para o desenvolvimento e a compreensão dos conceitos, modelos e teorias físicas estudados em sala de aula (ARAÚJO; ABIB, 2003). Entretanto, a sua utilização nas escolas costuma ser pouco frequente (SOUSA, 2005). As dificuldades relacionadas a determinados fatores, como carga horária da disciplina, tempo para preparo das atividades, falta de equipamentos e materiais e, na maioria dos casos, falta de um espaço adequado na escola para que tais práticas se desenvolvam, podem constituir os motivos pelos quais, muitas vezes, o professor deixa de utilizar as atividades experimentais como recurso didático durante as suas aulas.

Todavia, com o desenvolvimento das TIC no contexto educacional, a preocupação com novas possibilidades para o ensino também atingiu a questão da importância das práticas experimentais nas aulas de Ciências. Neste sentido, as simulações virtuais desenvolvidas sobre situações ou fenômenos que se desejam estudar constituem um recurso para auxiliar professores e alunos durante a abordagem de determinados conceitos científicos.

Entretanto, a utilização das simulações como estratégia para o ensino de Física não deve ser entendida como uma substituição aos experimentos reais, mas uma alternativa que visa enriquecer as situações desenvolvidas em sala de aula e contribuir para a aprendizagem dos temas abordados, de modo que o professor tem a possibilidade, inclusive, de combinar práticas experimentais reais com práticas baseadas nas simulações virtuais (PAZ, 2007; DORNELES; ARAÚJO; VEIT, 2012).

A razão pela qual as simulações não substituem os experimentos reside no fato de que toda simulação é uma maneira de expressar um modelo teórico. Mesmo que uma simulação inclua erros sistemáticos e estatísticos para torná-la mais próxima daquilo que efetivamente se mede no laboratório, esses erros não são experimentais, mas calculados a partir de um modelo.

Uma das contribuições associadas às simulações apontadas por Medeiros e Medeiros (2002) refere-se à visualização do fenômeno físico e dos elementos que ele envolve. Tal aspecto constitui um importante complemento à linguagem verbal e escrita durante o tratamento de um fenômeno e aos conceitos a ele relacionados. Para determinadas situações, nas quais a simulação permite a representação de elementos mais abstratos, essa importância adquire dimensão ainda mais relevante (MIRANDA et al., 2011). Também são muito úteis no caso de alguns experimentos difíceis ou mesmo impossíveis de se realizar, seja pela necessidade de equipamentos sofisticados, pelos riscos envolvidos ou mesmo pelo fato de envolverem intervalos de tempo muito curtos ou muito longos para a observação (FIOLHAIS; TRINDADE, 2003).

Deste modo, as simulações computacionais constituem um recurso em potencial que poderá trazer grandes contribuições para o ensino e a aprendizagem, na medida em que possibilitam a observação da situação virtual, a representação de um fenômeno cuja observação 
real a partir de um experimento não é viável ou não é possível de acontecer, dada a sua natureza.

Outro fator de destaque quanto às potencialidades das simulações diz repeito à interatividade por ela proporcionada, a partir de uma combinação de recursos tecnológicos, e ainda pela possibilidade de geração de diferentes animações, conforme a seleção pelo estudante dos parâmetros mais relevantes quanto ao que se pretende observar (MEDEIROS; MEDEIROS, 2002). Deste modo, propiciam aos alunos uma participação mais ativa ao longo das aulas, mudando assim a tradicional postura de meros ouvintes muitas vezes assumida por eles (GONÇALVES, 2005). Além disso, essa mudança no papel desempenhado pelo aluno durante sua aprendizagem é favorecida pelo fato das simulações exigirem dos estudantes respostas e tomadas de decisões, contribuindo para que o mesmo faça parte da construção de seu próprio conhecimento (FIGUEIRA, 2005).

Portanto, a simulação revela-se como um interessante recurso para o desenvolvimento de atividades que poderão contribuir quanto aos objetivos definidos para o ensino e a aprendizagem de conceitos físicos, uma vez que é capaz de favorecer situações que exigirão maior participação e envolvimento dos alunos durante o ensino. Tal fato é considerado significativo quando se parte do pressuposto de que um papel mais ativo dos estudantes durante as aulas é importante não apenas pela possibilidade de despertar maior interesse, mas também por poder proporcionar melhores resultados quanto a sua aprendizagem.

Ainda considerando a importância de uma atitude mais ativa por parte dos estudantes, nota-se também o potencial das simulações para o desenvolvimento de atividades de natureza investigativa. Tais atividades, conforme destaca Azevedo (2004), mais do que o entendimento de conceitos e conteúdos a respeito de um fenômeno físico, contempla também a aprendizagem de procedimentos e atitudes a partir da ação do estudante diante de um problema a ele apresentado. Conforme destaca a autora, a atividade investigativa, baseada na resolução de problemas, constitui um instrumento importante no desenvolvimento de habilidades e capacidades, como o raciocínio, a argumentação e a ação. A autora também ressalta que na "construção de um conhecimento, o processo é tão importante quanto o produto" (AZEVEDO, 2004, p.22).

De acordo com Zompero e Laburú (2011), não há um consenso entre os pesquisadores da área de Ensino de Ciências a respeito das atividades investigativas. Entretanto, observam os autores, apesar da polissemia verificada quanto ao uso do termo na literatura, nota-se que tais atividades são baseadas na resolução de problemas, exigindo do aluno "um papel intelectual mais ativo durante as aulas" (ZOMPERO; LABURÚ, 2011, p. 79). Deste modo, destacam os autores, as atividades investigativas exigem do aluno empenho para a sua realização, desenvolvimento de hipóteses, busca por informações para a resolução do problema proposto, comunicação dos estudos feitos para os demais colegas, promovendo, portanto, "a aprendizagem dos conteúdos conceituais, e também dos conteúdos procedimentais que envolvem a construção do conhecimento científico" (ZOMPERO; LABURÚ, 2011, p. 78). 
Assim, nota-se uma convergência entre as possibilidades oferecidas pelas simulações e os objetivos das atividades investigativas para a compreensão de conceitos e fenômenos da Ciência. Além de favorecer o desenvolvimento de habilidades cognitivas por parte do aluno, tais estratégias de ensino contribuem para que o estudante exerça um papel mais atuante durante o seu aprendizado.

Apesar das potencialidades associadas ao uso das simulações, é necessário que se considere também o fato de que a simulação representa a modelagem de um sistema físico e, portanto, envolve simplificações e aproximações da realidade, sendo esta sua principal diferença em relação aos experimentos reais (MEDEIROS; MEDEIROS, 2002). Diante disso, torna-se evidente a importância da presença e atuação do professor, o qual necessita não só ter em mente a simplificação da realidade trazida pela simulação e o exagero contido em alguns efeitos por ela apresentados, como também possibilitar que os alunos tomem conhecimento deste fato. Isto pode e deve ser feito no decorrer das discussões realizadas durante e após a execução das atividades com as simulações nas aulas. Além disso, a existência de tais aspectos relacionados às limitações das simulações deve ser considerada desde a escolha da simulação que será utilizada durante as aulas até o desenvolvimento das atividades que contemplarão seu uso.

Outro fator importante quanto às considerações realizadas diz respeito ao cuidado necessário para que o professor não passe a seus alunos a equivocada ideia de que a situação simulada representa evidências e provas para uma determinada teoria científica. "Uma simulação pode tão somente imitar determinados aspectos da realidade, mas nunca a sua total complexidade. Uma simulação, por isso, nunca pode provar coisa alguma” (MEDEIROS; MEDEIROS, 2002, p. 83).

Deste modo, tão importantes quanto as potencialidades oferecidas pelas simulações para o ensino de conceitos físicos são os seus limites aqui considerados. Da mesma forma que tais potencialidades não são suficientes para garantir que o aprendizado dos conceitos estudados de fato ocorra, a existência de limitações também não diminui os méritos das simulações enquanto recurso didático para as aulas de Física.

Retomando a ideia de visualização do fenômeno físico, proporcionada pelas simulações computacionais (MEDEROS; MEDEIROS, 2002; MIRANDA et al., 2011), apresentamos a seguir algumas considerações a respeito da sua importância para o ensino e a aprendizagem de conceitos físicos.

\section{Visualização e o Ensino de Física}

O termo visualização apresenta diversos sentidos e aplicações, nas diferentes áreas do conhecimento (GOBERT, 2005; PEREIRA, 2011). No caso específico das pesquisas em psicologia e educação, Gobert (2011) identifica três usos mais comuns: visualização externa, visualização interna e a visualização como habilidade espacial. A autora destaca ainda que estas três formas de uso para o termo não são mutuamente exclusivas, afirmando também que 
"a aprendizagem com uma visualização externa provavelmente requer que se construa uma representação mental interna do objeto ou fenômeno sob investigação e as habilidades de visualização espacial podem desempenhar um papel nesse processo de construção" (GOBERT, 2005, p. 74).

Dentre as formas citadas anteriormente, a visualização externa é considerada como sendo aquela mais utilizada para a aprendizagem (GOBERT, 2005), constituindo, no caso do ensino de ciências, representações que auxiliam o ensino e a aprendizagem de determinados temas e conceitos, como é o caso das representações feitas por gráficos, diagramas, modelos e, inclusive, as simulações, como aquelas geradas por OA.

De acordo com Uttal e O' Doherty (2008), a visualização externa é qualquer representação física que tem por objetivo tornar visível um conceito abstrato, tornando, assim, mais acessíveis durante a aprendizagem as informações de maior complexidade. Deste modo, a grande vantagem das visualizações externas consiste na sua capacidade em desenvolver o pensamento a partir do visual, em vez do abstrato e dos termos simbólicos. Percebe-se, no caso destes autores, um maior destaque para as representações em que se faz uso de imagens, como as fotografias, figuras, simulações etc.

Gilbert (2005), ao tratar das visualizações externas no ensino de ciências, destaca cinco modos de representação para o modelo de um fenômeno que está sendo estudado:

- Modo concreto ou material: representação tridimensional do modelo a partir da utilização de determinados objetos e materiais como ocorre na confecção de uma maquete, por exemplo.

- Modo verbal: descrição das entidades envolvidas na representação utilizada e das relações entre elas.

- Modo simbólico: representações por símbolos, fórmulas, equações e expressões, principalmente a partir da linguagem matemática.

- Modo visual: representações a partir de figuras, gráficos, animações etc.

- Modo gestual: movimento do corpo ou de uma de suas partes durante a explicação.

Além desses modos de representação, o autor aponta ainda três níveis para a visualização como representação externa:

- Nível Macroscópico: consiste na observação direta do fenômeno, como ocorre em uma experiência de laboratório, por exemplo, ou mesmo na observação de um fenômeno do cotidiano.

- Nível Sub-Microscópico: representação de entidades não visíveis e as relações que apresentam, como acontece no caso da representação de átomos, moléculas, íons etc.

- Nível Simbólico: representação simbólica das entidades sub-microscópicas a partir das relações quantitativas existentes entre elas, resultando em uma expressão matemática. 
A transição entre estes modos e níveis de representação torna-se necessária para que a visualização possa, de fato, contribuir para o aprendizado dos conceitos e dos temas estudados. Entretanto, trata-se de um processo que exige grande esforço intelectual do estudante (GILBERT, 2005; PEREIRA, 2011). Neste sentido, também é destacado o papel do professor durante o ensino, pois, além do esforço intelectual necessário, corre-se também o risco de que os estudantes possam "dirigir sua atenção apenas para a visualização em si, e não sobre o que ela pretende representar" (PEREIRA, 2011, p. 17).

Segundo Gobert (2005) a visualização também é utilizada para tratar das construções mentais internas, os denominados modelos mentais. Nesta perspectiva, a visualização envolve processos cognitivos, os quais implicam em "ver com os olhos da mente" (BOTZER; REINER, 2005; GOBERT, 2005).

De acordo com Rapp (2005) os modelos mentais são de difícil definição devido a uma série de razões. Dentre elas, cita a própria abstração que o conceito envolve e o fato de que também foi definido de maneira variada ao longo de diferentes pesquisas, de modo que, em sua maioria, se percebe pouca interação entre elas. Apesar disso, o autor caracteriza os modelos mentais, em um sentido mais geral, como sendo "organizações conceituais de informação na memória" (RAPP, 2005, p. 45). Para o autor, os modelos mentais constituem estruturas internas de um conhecimento organizado, as quais podem ser usadas para resolver problemas.

Para Moreira (1996), as pessoas não captam diretamente o que acontece no mundo exterior, mas constroem representações internas a respeito, ou seja, desenvolvem modelos mentais. Segundo o autor, um modelo mental constitui a "representação interna de informações que corresponde analogamente com aquilo que está sendo representado" (MOREIRA, 1996, p. 195). Nessa mesma perspectiva, Borges (1998) destaca que nossas explicações sobre aquilo que observamos são tentativas de compreender algo não familiar a partir daquilo que já estamos habituados, fazendo relações por meio de analogias. Além disso, destaca que o termo modelo mental é usado por alguns autores ao fazer referência às analogias que as pessoas usam ao tentar compreender conceitos e fenômenos físicos, mas também é comum a utilização do termo como um tipo próprio de representação interna do indivíduo.

No que diz respeito à Física, Botzer e Reiner (2005) ressaltam os processos cognitivos envolvidos nas práticas relacionadas a essa ciência e destacam a necessidade de se "ver com os olhos da mente", durante a realização de tais práticas. Assim, discute a ideia do pensar em imagens, processo que ocorre durante a visualização de um evento, a exploração mental de um diagrama ou comparando representações mentais pictóricas. Tal processo de pensamento por geração ou manipulação de imagem é tratado pelos autores por imagem mental ou, simplesmente, visualização.

Já a visualização como habilidade espacial está relacionada à capacidade de manipulação e transformação de imagens ou padrões espaciais (GOBERT, 2005). Trata-se, portanto, de um tipo de visualização que possui relação direta com as visualizações externas e internas, 
discutidas anteriormente, uma vez que a visualização requer a percepção e a manipulação mental de objetos no espaço (GILBERT, 2008).

Considerando a ideia de que os modelos na ciência podem ser vistos como uma simplificação do problema ou do fenômeno a ser estudado (GRECA; SANTOS, 2005), tal simplificação envolve uma redução progressiva, a partir da seguinte ordem: representações em três dimensões são reduzidas para duas dimensões e desta para uma dimensão (GILBERT, 2008). Neste sentido o contato do estudante com representações em três dimensões poderá acontecer em atividades de laboratório (nível macroscópico) ou ainda durante as explicações do professor a respeito de entidades do nível sub-microscópico, em que se faz uso dos modos concreto e gestual citados anteriormente.

Já o contato com as representações em duas dimensões também poderá ser realizado no laboratório, quando os alunos visualizam a projeção ou a secção transversal de elementos relacionados ao fenômeno estudado, mas também a partir de fotografias, figuras, gráficos, simulações virtuais etc. A representação em uma dimensão, por sua vez, diz respeito ao nível simbólico. Trata-se dos símbolos e equações utilizados pela ciência.

As considerações realizadas ao longo desta seção constituem elementos importantes ao analisarmos alguns dos problemas relacionados ao ensino e à aprendizagem de conceitos físicos. Na seção seguinte, descreveremos o contexto no qual a pesquisa foi realizada, assim como os procedimentos utilizados para o seu desenvolvimento.

\section{Contexto do estudo e procedimentos metodológicos}

A pesquisa realizada segue a abordagem qualitativa, a qual, além de ser descritiva, apresenta como características: o ambiente como fonte direta dos dados e o investigador como principal instrumento da pesquisa, além da preocupação voltada principalmente para o processo, mais do que para os resultados e o produto (LÜDKE; ANDRÉ, 1986; TIVIÑOS, 2009). Segundo Hammel (2010), ao se considerar a interpretação dos fenômenos e a atribuição de significados, a sala de aula se apresenta como um espaço propício para a realização da pesquisa qualitativa, considerando a necessidade do envolvimento direto do professor com os alunos, os sujeitos da pesquisa, durante a tarefa de estudo e análise do tema em seu ambiente e ao fazer a descrição dos fatos ali ocorridos.

Os estudos referentes a esta pesquisa foram realizados com 20 alunos da terceira série do Ensino Médio Regular, em uma escola pública estadual, localizada no interior do estado de São Paulo.

A investigação ocorreu ao longo de 10 aulas de Física, as quais foram desenvolvidas na sala de informática, local em que funciona o Programa Acessa Escola ${ }^{6}$. Embora o objetivo

\footnotetext{
${ }^{6}$ O Acessa Escola é "um programa do Governo do Estado de São Paulo, desenvolvido pelas Secretarias de Estado da Educação e de Gestão Pública, sob a coordenação da Fundação para o Desenvolvimento da Educação (FDE). Tem por objetivo promover a inclusão digital e social dos alunos, professores e funcionários das escolas
} 
principal desse programa não seja proporcionar um ambiente específico para o desenvolvimento de aulas por meio da utilização de recursos da informática, a existência de tal projeto na instituição torna isto possível, ainda que de forma limitada ${ }^{7}$.

Durante as aulas os alunos eram divididos em grupos de, no máximo, três alunos para a realização das sequências de atividades propostas. A cada grupo era entregue um CDROM contendo o OA utilizado, além de fichas apresentando as atividades que seriam realizadas, alguns questionamentos e também orientações que permitiram que os alunos fizessem registro de suas observações de um modo mais organizado. Os grupos receberam ainda um caderno para que pudessem, além dos registros de suas observações, fazer outras anotações sobre aquilo que consideraram pertinente em relação ao fenômeno e aos conceitos estudados.

Além dos materiais dos alunos descritos anteriormente, para a coleta de dados foram utilizados também o caderno de campo do professor-pesquisador, uma filmadora e um gravador de áudio. Outro instrumento de coleta de dados do qual se fez uso, ao longo do desenvolvimento da pesquisa, foram questionários para investigar a opinião dos alunos a respeito das aulas na sala de informática, em diferentes momentos da investigação, considerando principalmente aspectos relacionados aos processos de ensino e aprendizagem dos conceitos abordados, a partir das atividades propostas e desenvolvidas.

\section{As atividades desenvolvidas}

As atividades desenvolvidas para a utilização do OA são de natureza investigativa, entendidas como aquelas que possibilitam a elaboração de conjecturas e hipóteses, nas quais o aluno é o principal sujeito do processo. A partir das possibilidades de manipulação oferecidas pelo OA, foram elaboradas situações que pudessem favorecer o estudo dos conceitos eletromagnéticos envolvidos com base nas discussões e reflexões a respeito das observações realizadas em cada atividade ${ }^{8}$. Deste modo, as sequências de atividades buscaram estimular os estudantes quanto à argumentação e também à elaboração de hipóteses a respeito do fenômeno explorado a partir dos OA.

A sequência de atividades aqui apresentada constitui parte de um curso de Eletromagnetismo para o Ensino Médio. Deste modo, algumas discussões a respeito de outros conceitos e fenômenos eletromagnéticos aconteceram tanto antes da realização desta sequência como depois dela. Tais discussões também envolveram a abordagem investigativa, a partir do uso de simulações computacionais.

da rede pública estadual". (Fonte: <http://www.educacao.sp.gov.br/projetos/acessa-escola.> Acesso: 10 out. 2011.

${ }^{7}$ A sala possui cerca de 10 máquinas, de modo que esse pequeno número de computadores e a disposição física do ambiente, a qual apresenta os mesmos lado a lado em bancadas, algo semelhante a uma lan house, constituem fatores que dificultam a utilização do ambiente como sala de aula.

8 Essa sequência de atividades faz parte de um produto educacional desenvolvido no Programa de PósGraduação em Ensino de Ciências - Mestrado Profissional, da Universidade Federal de Itajubá (SOUSA, 2012). 
O OA Movimento de Cargas num Campo Magnético ${ }^{9}$ (Fig. 1) encontra-se disponível no Portal de Ensino de Ciências da USP, chamado Ciência à Mão. Trata-se de uma simulação computacional que permite investigar o comportamento de uma carga elétrica em movimento no interior de uma região de campo magnético que atua perpendicularmente à direção da trajetória da partícula em movimento.

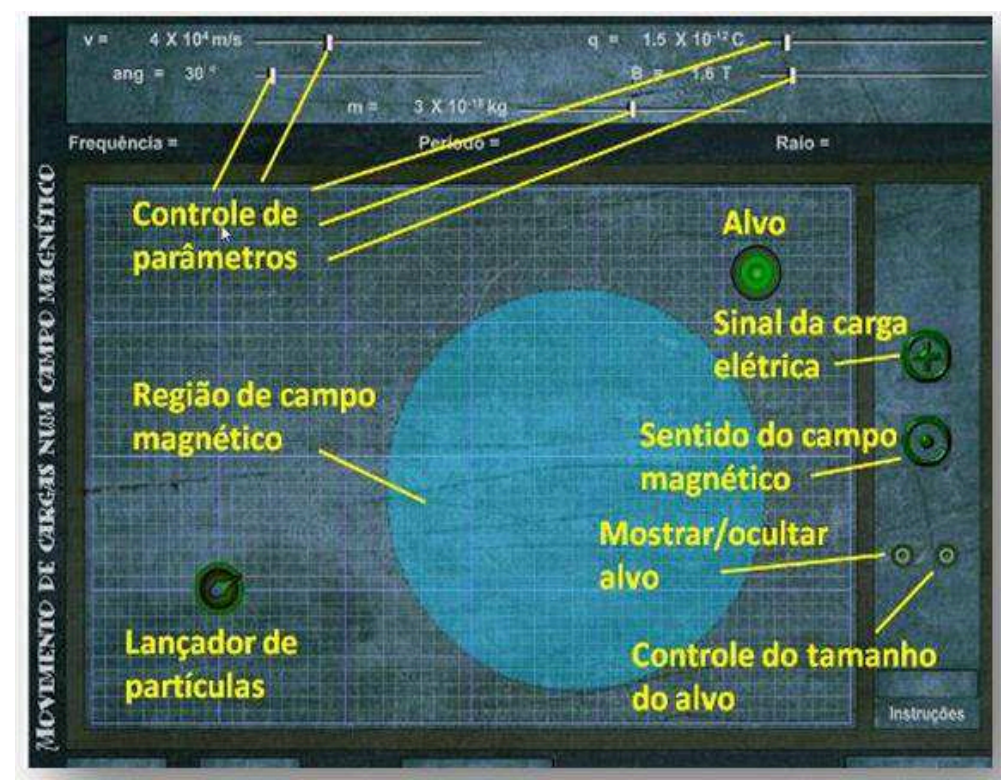

Fig. 1 -OA "Movimento de Cargas num Campo Magnético".

A partir do OA é possível simular a situação na qual um lançador dispara partículas carregadas para acertar um alvo, passando por uma região de campo magnético. A simulação possibilita que diversos parâmetros possam ser alterados ao se fazer o lançamento: ângulo de lançamento, valor da velocidade, massa da partícula, valor e sinal da carga elétrica da partícula, valor e sentido do campo magnético. Além da visualização da trajetória da partícula lançada, também é possível verificar o valor do raio da trajetória circular, assim como o período e a frequência do movimento circular uniforme executado pela partícula.

Nas sequências de atividades realizadas pelos alunos, foi possível inicialmente constatar a mudança da trajetória da partícula ao passar pela região de campo magnético. Em seguida, procurou-se evidenciar a trajetória retilínea fora da região de campo magnético e a trajetória circular no interior da região de campo. Posteriormente, a investigação desenvolveu-se a partir da observação da influência de alguns parâmetros no raio da trajetória circular da partícula.

A Tabela 1 apresenta as atividades desenvolvidas, a partir da utilização do OA, e os objetivos de cada uma delas.

\footnotetext{
9 <http://www.cienciamao.usp.br/tudo/exibir.php?midia=tex\&cod=_movimentodecargasnumcamp >. Acesso em: 15 mar. 2012
} 
Tabela 1 - Atividades realizadas com a utilização do OA "Movimento de Cargas num Campo Magnético".

\begin{tabular}{|c|c|}
\hline Atividade & Objetivo \\
\hline $\begin{array}{l}\text { Lançamentos de uma partícula para acer- } \\
\text { tar o alvo }\end{array}$ & $\begin{array}{l}\text { Perceber que a trajetória da partícula carregada é altera- } \\
\text { da ao passar por uma região de campo magnético atu- } \\
\text { ando perpendicularmente à direção do movimento. }\end{array}$ \\
\hline $\begin{array}{l}\text { Deslocamento da partícula fora da região } \\
\text { de campo magnético }\end{array}$ & $\begin{array}{l}\text { Observar que a trajetória da partícula fora da região de } \\
\text { campo magnético é sempre retilínea, mesmo alterando o } \\
\text { valor de sua carga elétrica. }\end{array}$ \\
\hline $\begin{array}{l}\text { Deslocamento da partícula no interior da } \\
\text { região de campo magnético }\end{array}$ & $\begin{array}{l}\text { Perceber a trajetória circular da partícula no interior da } \\
\text { região de campo magnético. }\end{array}$ \\
\hline Conclusões & $\begin{array}{l}\text { Escrever as conclusões iniciais a respeito do efeito do } \\
\text { campo magnético sobre a partícula em movimento. }\end{array}$ \\
\hline Influência da natureza da carga elétrica & $\begin{array}{l}\text { Perceber como o sinal da carga elétrica determina a sua } \\
\text { trajetória na região de campo. }\end{array}$ \\
\hline $\begin{array}{l}\text { Influência do sentido de atuação do campo } \\
\text { magnético }\end{array}$ & $\begin{array}{l}\text { Observar como o sentido do campo magnético influen- } \\
\text { cia a trajetória da partícula. }\end{array}$ \\
\hline $\begin{array}{l}\text { Influência da velocidade da partícula no } \\
\text { raio da trajetória circular }\end{array}$ & $\begin{array}{l}\text { Observar a dependência do raio da trajetória com o } \\
\text { valor da velocidade da partícula em movimento. }\end{array}$ \\
\hline $\begin{array}{l}\text { Influência da carga elétrica da partícula no } \\
\text { raio da trajetória circular }\end{array}$ & $\begin{array}{l}\text { Verificar a dependência do raio da trajetória com o } \\
\text { valor da carga da partícula em movimento. }\end{array}$ \\
\hline $\begin{array}{l}\text { Influência da intensidade do campo mag- } \\
\text { nético no raio da trajetória circular }\end{array}$ & $\begin{array}{l}\text { Observar a dependência do raio da trajetória com a } \\
\text { intensidade do campo. }\end{array}$ \\
\hline $\begin{array}{l}\text { Influência da massa da partícula no raio da } \\
\text { trajetória circular }\end{array}$ & $\begin{array}{l}\text { Verificar a dependência do raio da trajetória com o } \\
\text { valor da massa da partícula em movimento. }\end{array}$ \\
\hline $\begin{array}{l}\text { Questão } 1 \text { - Dependência do raio de cur- } \\
\text { vatura da trajetória em função das variá- } \\
\text { veis investigadas }\end{array}$ & $\begin{array}{l}\text { Organizar as conclusões obtidas a respeito dos parâme- } \\
\text { tros que influenciam a trajetória da partícula, conforme } \\
\text { as quatro últimas atividades realizadas. }\end{array}$ \\
\hline $\begin{array}{l}\text { Questão } 2 \text { - Dependência da intensidade } \\
\text { da força magnética em relação às variáveis } \\
\text { investigadas }\end{array}$ & $\begin{array}{l}\text { Estabelecer a relação entre a força magnética e os pa- } \\
\text { râmetros que influenciam a trajetória da partícula, a } \\
\text { partir da comparação com a expressão da força centrí- } \\
\text { peta. }\end{array}$ \\
\hline
\end{tabular}

A partir das observações registradas pelos alunos durante a realização das atividades, bem como das hipóteses e argumentos levantados por eles à medida que alguns questionamentos eram apresentados, foram desenvolvidas discussões em torno dos conceitos envolvidos na expressão da Força de Lorentz (HALLIDAY; RESNICK; WALKER, 2009). 
Para uma partícula portadora de carga q e com velocidade $\mathbf{v}$, na presença de um campo magnético B, observa-se a ação de uma força $\mathbf{F}$ que é expressa no SI por:

$$
\mathbf{F}=\mathrm{q}(\mathbf{E}+\mathbf{v} \times \mathbf{B})
$$

A simulação trata apenas do caso particular em que o campo elétrico $\mathbf{E}$ é nulo e a velocidade da partícula $\mathbf{v}$ é sempre perpendicular ao campo magnético $\mathbf{B}$. Nesse caso, a magnitude da Força de Lorentz é dada por

$$
F_{M}=q v B
$$

Nessa expressão, $q$ é a carga da partícula, $v$ é a magnitude de sua velocidade e $B$ a magnitude do campo magnético.

Deste modo, as atividades desenvolvidas tiveram como objetivo principal, considerando o seu caráter investigativo, fornecer subsídios para que os aspectos conceituais envolvidos em tal expressão pudessem ser estudados.

\section{Apresentação e análise de resultados}

Considerando a quantidade de dados gerados a partir dos diferentes instrumentos de coleta utilizados, a organização dos mesmos foi feita a partir das sequências de atividades aplicadas e da separação destas conforme os grupos que as realizaram.

A análise e a interpretação dos dados obtidos foram efetuadas a partir da triangulação, uma estratégia de análise apontada por alguns autores como sendo de grande valia para a pesquisa qualitativa por oferecer maior rigor e credibilidade em investigações dessa natureza (MOREIRA, 2009; TRIVIÑOS, 2009).

Durante o processo de análise de dados foi possível verificar, entre os temas convergentes, a questão da visualização. A análise que aqui realizamos considera a visualização do fenômeno físico durante a simulação proporcionada pelo OA. No caso das simulações feitas ao longo das atividades propostas, a visualização refere-se ao fato do estudante observar a trajetória de uma partícula com carga elétrica em movimento no interior de uma região de campo magnético e fora dela.

Conforme foi discutido na seção 2 deste trabalho, as simulações constituem um tipo de representação que auxilia o ensino e a aprendizagem de diversos conceitos e temas durante as aulas de Física e de Ciências, de modo geral. Durante a realização das sequências de atividades, tal fato pode ser constatado. De acordo com as considerações feitas por Gilbert (2005; 2008) a respeito das visualizações externas, o estudo dos conceitos com uso dos OA, nas sequências de atividades anteriormente descritas, envolve representações a partir do modo visual e no nível sub-microscópico.

A visualização proporcionada pelas simulações auxiliou os estudantes a pensar a partir do visual (UTTAL; O’ DOHERTY, 2008), e não a partir do simbólico e do abstrato, 
como é comum acontecer durante as aulas de Física, em especial, durante a abordagem do tema Eletromagnetismo (PAZ, 2007; PEREIRA, 2011).

O pensar a partir do visual pode ser verificado no registro dos alunos ao longo das atividades nas quais interagiram com o $\mathrm{OA}$ "Movimentos de cargas num campo magnético". Isto pode ser percebido já nas atividades iniciais, quando os alunos começam a observar que a partícula muda sua trajetória retilínea, ao passar pela região onde atua um campo magnético perpendicular a sua velocidade. $\mathrm{O}$ mesmo acontece nas atividades seguintes quando, a partir da visualização na simulação, os alunos puderam perceber a trajetória curvilínea descrita pela partícula na região de campo magnético.

A possibilidade de visualização da situação estudada também desempenhou um importante papel durante as discussões realizadas nas atividades que investigaram a dependência do raio da trajetória circular da partícula em relação a alguns parâmetros. Tal fato pode ser constatado, por exemplo, no caso da atividade em que se investigou a influência da carga elétrica da partícula em movimento. Nesta atividade, foram realizadas duas simulações nas quais apenas se alterou o sinal da carga elétrica em movimento. Algumas das respostas dos alunos ${ }^{10}$ para esta atividade podem ser verificadas a seguir:

Grupo 1: Carga positiva a curva será para baixo e depois sobe, a negativa não irá fazer uma volta completa e a curva será para cima.

Grupo 5: Observamos que o movimento realizado pela partícula de carga positiva realiza a entrada no campo magnético em linha reta, e após entrar no campo sai contornando a partícula de carga positiva. E quanto a partícula de carga negativa, entrou no campo magnético em linha reta e saiu em curva sem contornar a ela mesma. Uma outra observação foi que as duas partículas permaneceram com o mesmo valor no raio.

Grupo 7: A diferença observada entre os movimentos é que quando a partícula de carga é positiva, entra no campo magnético e faz uma curva, formando um círculo antes de sua retirada. Já quando a partícula tem carga negativa, ela segue sua trajetória em linha reta, ao ser lançada, e quando entra em contato com o campo magnético, ela é repelida.

No caso desta atividade, ficou clara a importância das visualizações, pois tornaram possíveis as comparações realizadas pelos estudantes ao relatarem suas conclusões. Tal fato pode ser evidenciado nas respostas dos alunos, principalmente naquelas em que eles fizeram uso de termos como "observamos que" e "a diferença observada é". O mesmo ocorreu na discussão desenvolvida em sala de aula para que os estudantes pudessem compreender que a

\footnotetext{
10 As respostas apresentadas são fiéis aos relatos originais dos alunos, obtidos nas fichas. Sendo assim, eventuais erros de português são transcritos sem correção. Além disso, em alguns relatos observou-se o uso inadequado de alguns termos pelos alunos, ao expressarem suas ideias, ou ainda algumas hipóteses incorretas a respeito do que era investigado. Neste caso, tais erros foram comentados e esclarecidos nas discussões desenvolvidas ao longo das aulas.
} 
única diferença quanto à influência da natureza das cargas em movimento é o sentido do movimento circular.

Alguns dados coletados a partir do questionário respondido pelos alunos também chamaram atenção quanto ao aspecto da visualização. Com o propósito de investigar a opinião dos estudantes a respeito das aulas de Física desenvolvidas na sala de informática da escola, eles responderam a um questionário com as seguintes perguntas: "Qual é a sua opinião a respeito da realização das aulas de Física na sala de informática? Você está gostando? Por que?"; "Comparando as aulas que têm sido realizadas na sala de informática com as aulas de Física na sala de aula convencional (quadro e giz e uso do caderno do aluno ${ }^{11}$ ), quais são as suas considerações a respeito? Cite os aspectos positivos e negativos." e "Em sua opinião, as atividades realizadas com o uso das simulações têm contribuído para a compreensão dos conceitos físicos estudados? De que forma?".

As respostas dos alunos quanto a esses questionamentos foram bastante positivas no que diz respeito ao uso do OA durante as aulas. De acordo com os mesmos, as aulas na sala de informática foram mais interessantes do que as aulas convencionais, pois, além de saírem da rotina, elas foram mais dinâmicas e divertidas. Nas respostas referentes à terceira questão, a visualização foi apontada como algo que contribuiu para o aprendizado dos conceitos estudados, como pode ser observado a seguir:

Aluno 1: [...] o simulador nos mostra melhor o funcionamento dos conceitos físicos do que uma imagem em um livro.

Aluno 2: Você aprende observando e fazendo as simulações, você pode ver o que está estudando.

Aluno 3: [...] quando visualizado é possível compreender melhor, pois é possível observar como acontece e não só imaginar.

As respostas dos alunos aqui apresentadas foram obtidas após as primeiras aulas nas quais a pesquisa se desenvolveu. Nas aulas finais, os alunos responderam a um novo questionário, o qual, além de apresentar as mesmas questões do primeiro, solicitava aos alunos que também manifestassem suas impressões, críticas e sugestões sobre a experiência de aula vivenciada. Novamente, os alunos se manifestaram de forma positiva quanto às aulas e mencionaram a visualização como algo que contribuiu para o entendimento dos conceitos estudados:

Aluno 2: [...] na maneira de que você participa da aula, vendo as simulações, você vê o que está aprendendo.

Aluno 3: [...] visualizando, podemos movimentar, trocar valores e vermos a diferença com os efeitos realizados pelo simulador, diferente de ouvir e não poder observar em sala de aula.

\footnotetext{
11 Trata-se do material fornecido pela Secretaria de Educação do Estado de São Paulo, contendo textos e atividades para serem desenvolvidos durante as aulas.
} 
Aluno 4: [...] pois uma coisa explicada sem demonstrações é mais difícil de imaginar e entender, por isso o uso do simulador contribui bastante na compreensão dos estudos físicos.

Nas respostas apresentadas é possível perceber que os alunos consideram ser mais fácil entender o que está sendo estudado pelo fato da simulação permitir "ver” o que está sendo estudado em vez de ter que imaginar. Tal fato vai ao encontro das ideias sobre o pensar a partir do visual, já mencionadas no início desta seção.

De acordo com Pereira (2011), a visualização a partir da representação gerada diretamente pela simulação no OA pode facilitar o entendimento dos conceitos estudados, na medida em que existe a possibilidade de se poupar esforços dos alunos quanto às manipulações mentais necessárias para imaginar o fenômeno, de modo que tais manipulações possam ser dedicadas exclusivamente para a compreensão dos conceitos envolvidos. Isto se torna mais significativo, principalmente, no caso dos conceitos com maior nível de dificuldade e abstração, como foi o caso dos conceitos eletromagnéticos estudados a partir das atividades desenvolvidas.

Considerando ainda a importância atribuída pelos alunos à visualização do fenômeno durante seu estudo, duas respostas chamaram a atenção por corroborar as ideias de alguns autores discutidas nas seções anteriores. Entre as respostas do primeiro questionário, um dos alunos afirma que "o simulador nos mostra melhor o funcionamento dos conceitos físicos do que uma imagem em um livro". O termo "mostra melhor", citado por ele, provavelmente faz referência à limitação, no caso dos livros, de uma representação estática para fenômenos dinâmicos, o que não acontece no caso das simulações, considerando as animações interativas que elas apresentam (MEDEIROS; MEDEIROS, 2002).

Já no segundo questionário, uma aluna afirma que "visualizando, podemos movimentar, trocar valores e vermos a diferença com os efeitos realizados pelo simulador, diferente de ouvir e não poder observar em sala de aula". Deste modo, além de mencionar o papel da visualização como complemento à linguagem verbal (MEDEIROS; MEDEIROS, 2002), a aluna também faz referência à questão da interatividade relacionada à possibilidade de alterar parâmetros, o que dá uma importante contribuição para a compreensão dos conceitos estudados (MEDEIROS; MEDEIROS, 2002; MIRANDA et al., 2011).

Portanto, para os estudantes, o uso do OA constituiu um elemento colaborador durante as aulas de Física.

\section{Considerações finais}

Nesse artigo, descrevemos o desenvolvimento de práticas investigativas no Ensino de Física, a partir do uso de um AO para o estudo da Força de Lorentz. Nas situações desenvolvidas em sala de aula, a partir da realização das atividades com a utilização de um OA, a visualização do fenômeno físico estudado destacou-se como uma interessante contribuição 
para o ensino da Física. As visualizações parecem auxiliar os alunos quanto ao entendimento dos conceitos estudados, à medida que podem facilitar a compreensão de elementos mais abstratos relacionados a estes conceitos. O desenvolvimento de habilidades relacionadas à capacidade de abstração é considerado importante durante o estudo da Física e das Ciências de modo geral. Entretanto, uma representação mais concreta obtida nas simulações não traz prejuízos quanto a estas habilidades, mas, pelo contrário, contribui para que as mesmas sejam desenvolvidas, auxiliando os estudantes na superação dos obstáculos iniciais quanto a isto.

As situações vivenciadas ao longo da investigação realizada mostraram que, além das contribuições para o ensino de Física, outros fatores bastante significativos e que merecem ser destacados foram a participação e o envolvimento dos alunos durante as aulas. A mudança na dinâmica das aulas, proporcionada pela elaboração de atividades que contemplaram a utilização do OA, possibilitou que os alunos assumissem um papel mais ativo durante o estudo do Eletromagnetismo. Isto ocorreu não apenas durante a execução das atividades, como também nas discussões que se desenvolveram a respeito dos conceitos estudados.

Mesmo nos momentos em que uma exposição maior do professor a respeito dos conceitos e conteúdos se fez necessária, foi possível estabelecer o diálogo com os estudantes durante as explicações, algo muitas vezes raro em nossas aulas de Física. É fato que nem todos os alunos se envolveram da mesma forma, principalmente nas discussões desenvolvidas, e, muitas vezes, foi necessária a intervenção maior do professor na tentativa de que isto ocorresse. Do mesmo modo, nem todas as aulas ocorreram da mesma maneira, sendo também necessário para algumas delas um esforço maior para conseguir a atenção dos alunos. De qualquer maneira, ainda que em proporções diferentes, mudanças positivas em relação à postura dos estudantes puderam ser observadas.

Ademais, embora o foco da pesquisa fosse as contribuições e as potencialidades dos OA para o ensino de conceitos sobre Eletromagnetismo, alguns elementos relacionados à aprendizagem também puderam ser observados nos dados obtidos ${ }^{12}$. Entretanto, considera-se a aprendizagem um dos objetivos do ensino, independente do fato da mesma ser ou não alcançada. Sendo assim, possíveis contribuições do uso de OA para o aprendizado dos conceitos estudados não podiam ser descartadas e, deste modo, também são consideradas na análise e na interpretação dos resultados.

\section{Referências bibliográficas}

ALMEIDA, C. Z.; VIEIRA, M. B.; LUCIANO, N.A. Ambiente Virtual de Aprendizagem: uma proposta para autonomia e cooperação na disciplina de informática. In: SIMPÓSIO BRASILEIRO DE INFORMÁTICA NA EDUCAÇÃO, 12. Anais... Vitória, SBIE, 2001.

\footnotetext{
12 Uma discussão a respeito dos significados para os termos ensino e aprendizagem e as possíveis inter-relações que envolvem, ainda que relevante, foge do escopo deste trabalho.
} 
ARAÚJO, I. S.; VEIT, E. A. Uma Revisão da Literatura Sobre Estudos Relativos a Tecnologias Computacionais no Ensino de Física. Revista Brasileira de Pesquisa em Ciências, v. 4, n. 3, p. 5-18, 2004.

ARAÚJO, M. S. T.; ABIB, M. L. V. S. Atividades experimentais no ensino de física: diferentes enfoques, diferentes finalidades. Revista Brasileira de Ensino de Física, v. 25, n. 2, p. 176-194, 2003.

AZEVEDO, M. C. P. A. Ensino por investigação: problematizando as atividades em sala de aula. In: CARVALHO, A. M. P. (Org) Ensino de ciências: unindo a pesquisa e a prática. São Paulo: Thomson, 2004, p. 19-33.

BORGES, A. T. Modelos Mentais de Eletromagnetismo. Caderno Catarinense de Ensino de Ensino de Física, v. 15, n. 1, p. 7-31, 1998.

BOTZER, G.; REINER, M. Imagery in physics: From physicists' practice to naïve students'learning. In J. K. Gilbert (Ed.). Visualization in Science Education. Dordrecht: Springer, 2005, p. 147-168.

DORNELES, P. F. T.; ARAUJO, I. S.; VEIT, E. A. Integração entre atividades computacionais e experimentais como recurso instrucional no ensino de Eletromagnetismo em Física Geral. Ciência \& Educação, v. 18, n. 1, p. 99-122, 2012.

FIGUEIRA, J. S. Easy Java simulations: Modelagem computacional para o ensino de Física. Revista Brasileira de Ensino de Física, v. 27, n. 4, p. 613-618, 2005.

FIOLHAIS, C.; TRINDADE, J. Física no computador: o computador como uma ferramenta no ensino e na aprendizagem das ciências físicas. Revista Brasileira de Ensino de Física, v. 25, n. 3, p. 259-272, 2003.

GILBERT, J. K. Visualization in science education. Dordrecht, Netherlands: Springer, 2005.

GILBERT, J. K. Visualization: Theory and Practice in Science Education. Dordrecht, The Netherlands: Springer, 2008.

GOBERT, J. D. Grasping leveraging technology and cognitive theory on visualization to promote students' learning. In: GILBERT, J. K. (Ed.). Visualization in Science Education. Dordrecht: Springer, 2005. p. 73-90.

GONÇALVES, L. J. Uso de animações visando a aprendizagem significativa de Física Térmica no Ensino Médio. 2005. 97 f. Dissertação (Mestrado Profissionalizante em Ensino de Física) - Universidade Federal do Rio Grande do Sul, Porto Alegre.

GRECA, I. M.; SANTOS, F. M. T. Dificuldades da Generalização das estratégias de Modelação em Ciências: o caso da Física e da Química. Investigação em Ensino de Ciências, v. 10, n. 1, p. 31-46, 2005.

HALLIDAY, D.; RESNICK, R.; WALKER, J. Fundamentos de Física. 8. ed. Rio de Janeiro: LTC, 2009. v. 3.

HAMMEL, A. Uso de um hipertexto baseado em simulação e vídeo no ensino dos conceitos de calor e temperatura em nível médio. 2010. 117 f. Dissertação (Mestrado Profissionalizante em Ensino de Física e Matemática) - Centro Universitário Franciscano, Santa Maria.

LÜDKE, M.; ANDRÉ, M. E. D. A. Pesquisa em Educação: abordagens qualitativas. São Paulo: E.P.U., 1986. 
MARTINS, A. A.; GARCIA, N. M. D.; BRITO, G. S. O Ensino de Física e as Novas Tecnologias de Informação e Comunicação: uma análise da produção recente. In: SIMPÓSIO NACIONAL DE ENSINO DE FÍSICA, 19, 2011, Manaus. Anais... Manaus: SNEF, 2011.

MEDEIROS, A.; MEDEIROS, C. F. Possibilidades e limitações das simulações computacionais no ensino da Física. Revista Brasileira de Ensino de Física, v. 24, n. 2, p. 77-86, 2002.

MIRANDA, M. S.; ARANTES, A. R.; STUDART, N. Objetos de Aprendizagem no Ensino de Física: usando simulações do PhET. In: SIMPÓSIO NACIONAL DE ENSINO DE FÍSICA, 19, 2011, Manaus. Anais... Manaus: SNEF, 2011.

MOREIRA, C. A. O. Ambiente virtual interativo no ensino de Ciências: uma abordagem sociocultural. 2009. 153 f. Dissertação (Programa de Pós-Graduação Interunidades em Ensino de Ciências) - Universidade de São Paulo, São Paulo.

MOREIRA, M. A. Modelos Mentais. Investigações em Ensino de ciências, v. 1, n. 3, p. 193 232, 1996.

PAZ, A. M. Atividades experimentais e informatizadas: contribuições para o ensino de eletromagnetismo. 2007. 228 f. Tese (Doutorado em Educação Científica e Tecnológica) Universidade Federal de Santa Catarina, Santa Catarina.

PEREIRA, J. A. Fenômenos Eletromagnéticos e sua visualização: um obstáculo de aprendizagem. 2011. 164 f. Dissertação (Mestrado em Educação Científica e Tecnológica) Universidade Federal de Santa Catarina, Florianópolis.

RAPP, D. Mental Models: Theoretical issues for visualizations in science education. In: GILBERT, J. K. (Ed.). Visualization in Science Education. Dordrecht: Springer, 2005, p. 43-60.

ROSA, P. R. S. O uso de computadores no ensino de Física. Parte I: Potencialidades e Uso Real. Revista Brasileira de Ensino de Física, v. 17, n. 2, p. 182-195, 1995.

SANTOS NETO, E. S.; SASSERON, L. H.; PIETROCOLA, M. "Como usar objetos de aprendizagem" em aulas de Ciências: articulações entre teoria e prática na formação à distância de multiplicadores. Experiência em Ensino de Ciências, v. 4, n. 2, p. 83-92, 2009.

SOUSA, J. M. Objetos de aprendizagem e o ensino de conceitos de eletromagnetismo no Ensino Médio. 2012. 177f. Dissertação (Mestrado Profissional em Ensino de Ciências) Universidade Federal de Itajubá, Itajubá.

SOUSA, J. M. O perfil do ensino de Física do ensino médio na cidade de Itajubá: Fundamentos para a Licenciatura. 2005. 67 f. Trabalho de conclusão de curso (Física Licenciatura) - Universidade Federal de Itajubá, Itajubá.

TRIVIÑOS, A. N. Introdução à Pesquisa em Ciências Sociais. São Paulo: Atlas, 2009.

UTTAL, D. H.; O' DOHERTY, K. Comprehending and learning from 'visualizations': a developmental perspective. In. GILBERT, J. K. (Ed.). Visualization: theory and practice in Science Education. Dordrecht: Springer, 2008. p. 53-72.

ZOMPERO; A. F.; LABURÚ, C. E. Atividades investigativas no ensino de ciências: aspectos históricos e diferentes abordagens. Revista Ensaio, v. 13, n. 3, p. 37-80, 2011. 